\title{
Dernière ligne droite pour la révision de la LPTh
}

\section{Ernst Gähler}

Dr, vice-président de la FMH, responsable du département Tarifs et conventions pour la médecine ambulatoire en Suisse / Professions paramédicales

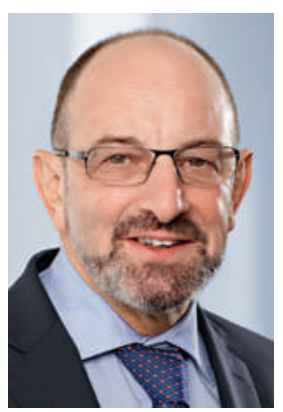

En mettant en consultation son projet de révision de la loi sur les produits thérapeutiques (LPTh) en 2009, l'ancien conseiller fédéral Pascal Couchepin a souhaité supprimer ou tout du moins limiter la remise de médicaments par les médecins (propharmacie), au motif que ce système créerait des incitatifs erronés et générerait des coûts plus élevés. Selon lui, les médecins dispensants seraient incités à prescrire davantage de médicaments soumis à ordonnance, d'où la nécessité d'interdire ce système. Par ailleurs, la propharmacie coûterait plus cher que le canal traditionnel des pharmacies.

Désormais, les pharmaciens pourront délivrer eux-mêmes des médicaments soumis à ordonnance sans pour autant disposer de la formation nécessaire.

Ce projet a soulevé une telle indignation que l'ancien chef du DFI avait dû relativiser ses propos après quelques heures seulement. En réalité, aucune étude ne prouve à ce jour que les médecins dispensants sont incités à remettre davantage de médicaments pour des raisons économiques. Au contraire, certaines études scientifiques relèvent qu'ils prescrivent plus de médicaments génériques que leurs confrères et contribuent donc massivement à réduire les coûts de santé. Par ailleurs, les statistiques de santésuisse montrent que les coûts de médicaments par personne sont moins élevés dans les 17 cantons suisses alémaniques qui pratiquent la propharmacie que dans les cantons qui ont recours à des ordonnances. Sans oublier que dans ces derniers, il faut encore tenir compte de la rémunération basée sur les prestations (RBP) que les pharmaciens peuvent exiger et qui vient encore augmenter les coûts de ce canal. L'Office fédéral de la santé publique (OFSP) a certainement eu connaissance de ces chiffres puisque le projet de révision de la loi sur les produits thérapeutiques du conseiller fédéral Alain Berset ne prévoit plus de limitation régionale de la propharmacie. En revanche, il fait un autre pas en direction des pharmaciens. D'une part en les autorisant à remettre les médicaments soumis à ordonnance de la liste B sans ordonnance préalable d'un médecin, bien qu'ils ne disposent pas de la formation nécessaire dans la pose de diagnostic, et d'autre part en obligeant les médecins dispensants à fournir une ordonnance à leurs patients lors de chaque remise de médicament afin que ceux-ci puissent, s'ils le souhaitent, renoncer à retirer leurs médicaments auprès de leur médecin et se rendre à la pharmacie.

Selon les estimations de la FMH, cette contrainte bureaucratique - en plus de partir du principe que le patient n'est pas assez «mature» ni indépendant pour exprimer son propre avis - générerait une hausse des coûts à hauteur de 100 à 150 millions de francs par année à la charge des assureurs. Une aberration, sachant que ces coûts supplémentaires n'offriraient aucune plus-value sociale ou économique. En outre, les médecins seraient tenus de consacrer une partie encore plus grande de leur temps aux tâches administratives au détriment du temps passé auprès de leurs patients, alors que nous nous trouvons actuellement en pénurie de médecins de famille.

Les assurés devraient payer jusqu'à 150 millions de francs supplémentaires par an si les médecins dispensants étaient obligés de fournir une ordonnance à chaque fois qu'ils remettent un médicament.

J'espère donc vivement que dans cette dernière ligne droite de la révision de la loi sur les produits thérapeutiques, les Chambres fédérales se raviseront et renonceront à cette obligation inutile, coûteuse et bureaucratique. Cette loi a été créée pour protéger la population contre d'éventuels risques sanitaires liés aux médicaments. L'obligation faite aux médecins dispensants de délivrer une ordonnance n'a donc rien à y faire. 